\title{
Haptic Communication for a 2D Pointing Task in a Virtual Environment
}

\author{
Boris Takač ${ }^{1}$, Amine Chellali ${ }^{2}$, Cédric Dumas ${ }^{3}$, Isabelle Milleville ${ }^{1}$, Cristol \\ Grosdemouge $^{4}$, Caroline G.L. Cao ${ }^{4}$ \\ ${ }^{1}$ Institut de Recherche en Communications et Cybernétique de Nantes \\ ${ }^{2}$ Ecole des Mines de Nantes \\ ${ }^{3}$ Commonwealth Scientific and Industrial Research Organisation \\ ${ }^{4}$ Tufts University
}

\begin{abstract}
This paper examines the properties of haptic communication between two human operators using kinesthetic haptic devices in a collaborative task in a virtual environment. Twenty subjects, divided into 10 dyads, participated in a 2D pointing task. Each dyad consisted of a supervisor and an acting agent. The supervisor's role was to guide the acting agent towards a target in the virtual environment through either verbal or haptic communication only. Verbal communication was found to be the most efficient means of communication, but collaboration was also effective using haptic communication. Several different haptic communication strategies were observed, all with equal effectiveness as measured by task completion time. These strategies followed the same pattern as the verbal strategies. These results suggest that haptic communication in a virtual environment is possible, allowing for future designs of haptically enhanced collaborative work in virtual environments.
\end{abstract}

\section{INTRODUCTION}

Collaborative Virtual Environments (CVEs) are virtual reality spaces that enable participants to collaborate and interact with common objects as if physically co-located. These environments usually aim to provide users with a sense of realism by incorporating realistic 3D graphics, spatial sounds, and in some cases haptic devices. Haptics in CVEs have been used in many applications, such as medicine (e.g., surgical simulators), design (e.g., CAD systems) and education (e.g., mentor-guided motor skill learning).

When manipulating objects in CVE, the haptic modality can be crucial for understanding the use of the system, and on other occasions, when the audio and visual modalities are already engaged in communication, haptic feedback may be an additional channel of communication. To enable intuitive physical/haptic interaction in CVE, an understanding of natural haptic interaction between humans is important when developing such systems (Chellali, Dumas, \& Milleville, 2010).

Previous investigations of haptics in CVE included the examination of social interaction benefits of haptics in CVE (Ho, Basdogan, Slater, Durlach, \& Srinivasan, 1998); application of Fitts' law for human dyad task performance (Reed, Peshkin, Colgate, \& Patton, 2004), and kinesthetic interactions (Reed, Peshkin, Hartmann, Colgate, \& Patton, 2005). Kinesthetic interaction experiments spurred the Specialization hypothesis for dyads. The theory of Specialization suggests that each member of the dyad should specialize in executing certain parts of a task, while synchronizing control of the task through haptic communication. The nature of haptic communication and the cues used for indicating control transition points were left undisclosed. Haptic modality as a possible communication channel was considered by Chan, MacLean, \& McGrenere (2008). However, in these studies, haptic signals to be used by subjects were prescribed, without regard for the natural way in which humans develop haptic messages on their own.
This study investigates the ways in which haptic communication through kinesthetic haptic devices can be established and used to exchange information about the ongoing collaborative task between two participants.

An experiment was conducted using a pair of kinesthetic haptic devices in a collaborative task in CVE. The objective of the experiment was to answer the following questions:

1. Can two collaborators working in a virtual environment communicate through haptic devices?

2. If "yes", how can this communication be analyzed and interpreted?

\section{METHODS}

\section{Virtual Environment}

A collaborative virtual environment, which included a haptic link between two remote participants, was designed (Figure 1). A haptic link is defined as the connection between two identical haptic devices such that they directly copy each other's movements. The haptic link is effective when translations, rotations, velocities and forces produced by one device are reproduced exactly by the other device.

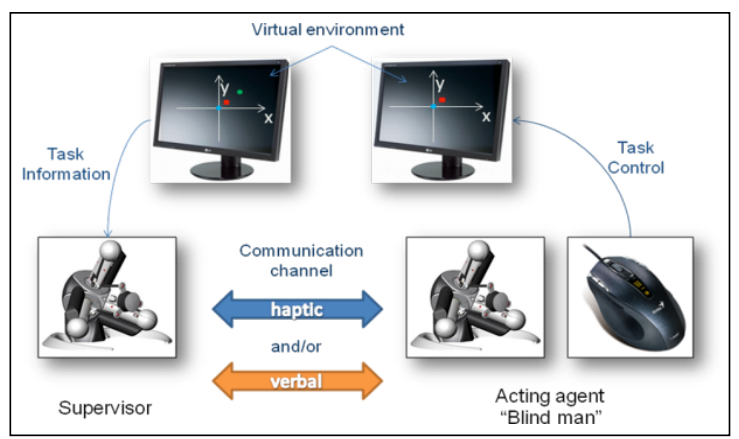

Figure 1: Haptic link between the input devices and the shared virtual environment. 
The devices used in this experiment were the Virtuose $6 D$ Desktop from Haption, a fully parallel robot with three legs and a platform. The actual work volume of the device is a sphere $12 \mathrm{~cm}$ in diameter, as shown in Figure 2. The device has a spatial resolution of $1.5 \times 10^{-2} \mathrm{~mm}$.

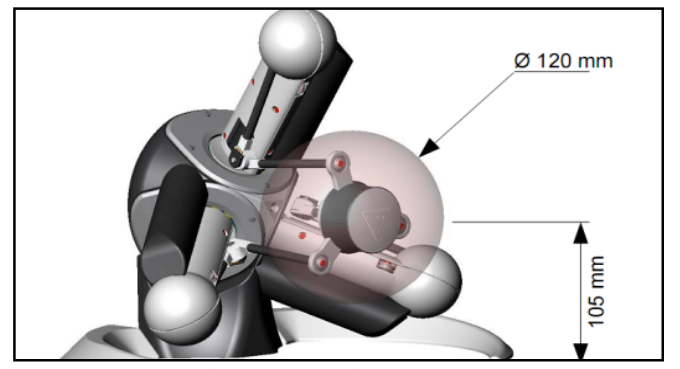

Figure 2: Workspace of the Virtuose 6D Desktop.

Virtual environment and task design. A simple collaborative task consisting of two separate components was designed. The task required target position information that could only be acquired visually, and pointing action that could only be executed through motor input control. A 2D virtual task environment was chosen to limit the complexity of data analysis while maintaining a high degree of task difficulty. A pointing task was chosen, and modeled after Fitts' task.

The virtual environment was designed using OpenGL, and consisted of a dark computer screen with a blue starting position, a red cursor, and a green target (Figure 3, Left). This view of the virtual environment was provided to the Supervisor of a dyad, whose job was to guide the Acting Agent in the dyad to the green target through either verbal or haptic communication. The Supervisor had visual information about the target location, but no control over the cursor. The Acting Agent had a mouse that controlled the cursor, but his/her view of the virtual environment lacked the presence of the target and thus must rely on the Supervisor's guidance to move the cursor to the target position (Figure 3, Right). The target location was randomly presented in one of 16 locations on the screen in each trial. Depending on the location of the target, the pointing task varied in the Index of Difficulty.

Fitts' law for two people collaborating on a simple manual task with identical targets has been validated (Reed et al., 2004). The Index of Difficulty (ID) can be calculated using the formulation of Fitts' law:

$$
I D=\log _{2}\left(1+\frac{D}{W}\right)
$$

where $\mathrm{D}$ is the distance from the starting point to the center of the target, also known as the amplitude of the movement, and $\mathrm{W}$ is the width of the target measured along the axis of motion. ID values were chosen by varying distances $\mathrm{D}$ (in screen units), keeping $\mathrm{W}$ constant and respecting the following constraints: a) absolute linear displacement between consecutive distance values must be greater than 0.2 screen units, b) keep the same increment between ID values, when possible. Given the size of the monitor and the centered starting position for the pointing task, we obtained 16 different target locations with different
IDs: a) ID1 (ID=1.8, $\mathrm{D}=0.2$ ), b) ID2 (ID=2.8, $\mathrm{D}=0.45$ ), c) ID3 $(\mathrm{ID}=3.3, \mathrm{D}=0.7), \mathrm{d})$ ID4 (ID=3.8, $\mathrm{D}=0.97)$. Each ID consisted of 4 different target positions in the CVE.

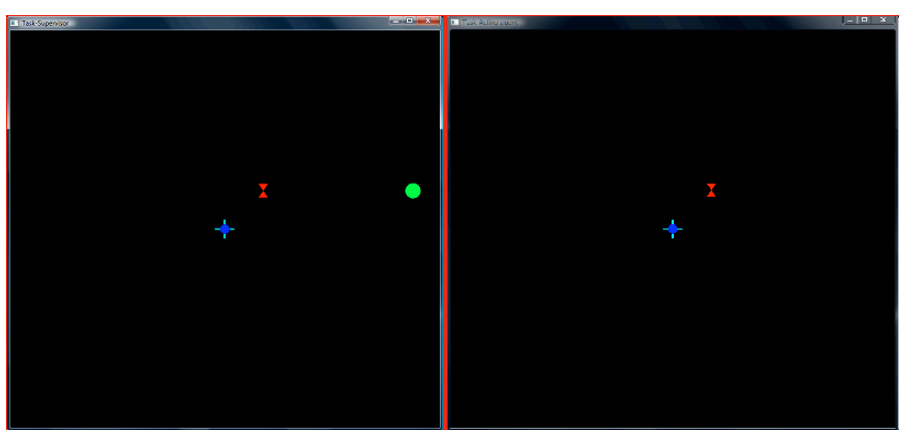

Figure 3: Virtual environment for the pointing task. Left, supervisor interface. Right, acting agent interface.

\section{Experimental Design}

Participants. Twenty subjects aged 19-55 participated in the study. There were 17 male (2 left handed) and 3 female (1 left handed) volunteers. None of the participants had experience with either virtual environments or with haptic devices. They were randomly paired to form 10 dyads.

Experimental Protocol. At the beginning of each session, members of the dyad performing the experiment were allowed to choose their workstation without having prior knowledge about what they would have to do. The dyad member who sat first at the workstation for the Acting agent role was labeled "Participant A", while his/her partner was the de facto Supervisor and labeled "Participant B".

Participants were told that the experiment was about haptic communication, and that they had to collaborate using haptic devices only (without talking) or verbally (in French or English) in order to complete a simple task. Then, the collaborative pointing task was presented, where both participants were able to see each other's screens simultaneously and experienced the behavior of the linked haptic devices.

In the verbal communication condition, the Supervisor used words, such as "move cursor to the right..." while the Acting Agent manipulated a mouse to move the cursor on the screen according to instruction. In the haptic communication condition, the Supervisor and the Acting Agent each held a haptic device. The Supervisor used the haptic device to "push" the Acting Agent's hand as felt through the haptic link. This force feedback was then translated by the Acting Agent to move a mouse held in the other hand.

Participants performed 4 practice trials of the task with full visual information, along with haptic communication. However, they were not allowed to talk about future strategy they could use. During the test trials, a curtain was drawn between the participants to prevent visual feedback from the other monitor, as well as from each other's body language (Figure 4). Subjects performed the task in four blocks, each block consisting of 16 randomly presented targets. Two 
blocks were performed using haptic communication, while the other two were performed using verbal communication.

Subjects switched roles after each block of trials. The order of communication conditions was counterbalanced. All sessions were filmed with a digital camera for off-line analysis.

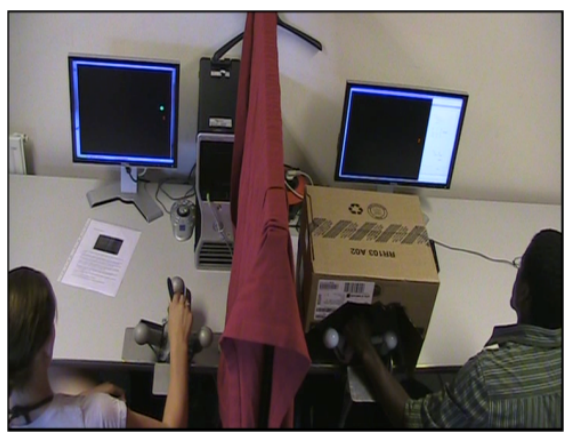

Figure 4: Experimental setup.

Variables. There were three independent variables in this experimental design: communication modality (2), order of communication conditions (2), and the task ID (4). The dependent variables were task completion time, output kinematics of the haptic devices, and verbalizations.

\section{RESULTS}

\section{Haptic Performance}

Time to task completion was analysed using analysis of variance at $\alpha=.05$.

There was a significant effect of order $(F(1,622)=$ $11.121, p<.001)$, showing that participants who started with the haptic modality were overall less effective $(\mathrm{T}=21623 \mathrm{~ms})$ than participants who started with verbal modality $(\mathrm{T}=$ $15395 \mathrm{~ms})$. There was also a significant effect of the communication modality $(F(1,622)=68.333, p<.001)$ where performance using verbal communication was faster $(\mathrm{T}=$ $11264 \mathrm{~ms})$ than using haptic communication $(=25799 \mathrm{~ms})$. The task ID also had a significant effect on time to task completion $(F(3,622)=5.570, p<.001)$. The task took longer when the ID was higher. There was a significant interaction effect between order and communication modality $(F(1,622)=$ $8.186, p<.001)$. Performance in the verbal condition was equally well regardless of which order the subjects were in. However, those who switched to the haptic condition after experience with verbal communication had a large decrement in performance in the haptic condition, taking much longer to perform the task $(\mathrm{T}=31641 \mathrm{~ms})$ than those who started with the haptic condition $(\mathrm{T}=19957 \mathrm{~ms})$ (Figure 5).

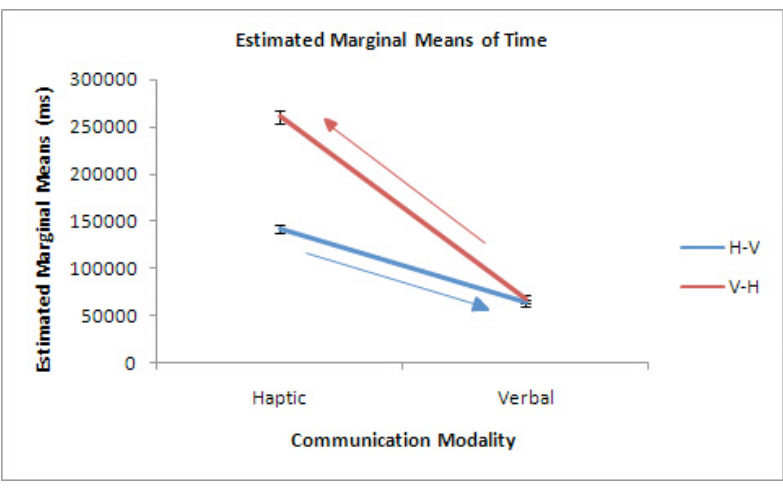

Figure 5: Task completion time as a function of the order and the communication modality.

\section{Haptic communication strategies}

By analyzing the output from the haptic devices, three haptic communication strategies were observed:

Motion Copying. Motion Copying seemed to be the most natural and the most obvious way to transmit spatial information through the haptic link. The recordings showed that each dyad started its haptic portion of the session by using this method. The participants were using Motion Copying if:

a) The supervisor used continuous movements inside the active workspace of the haptic device

b) Haptic device movements were changing concurrently along both axes of the work plane

c) The acting agent responded with mouse movements that tried to copy the trajectory of the haptic device

d) The mouse trajectory was continuous and was changing concurrently along both axes.

This strategy seemed to be effective only if the haptic devices were operated inside the active workspace. This means that the strategy usually worked for target points with IDs of 1.8 and 2.8. An example of Motion Copying is shown inn Figure 6.

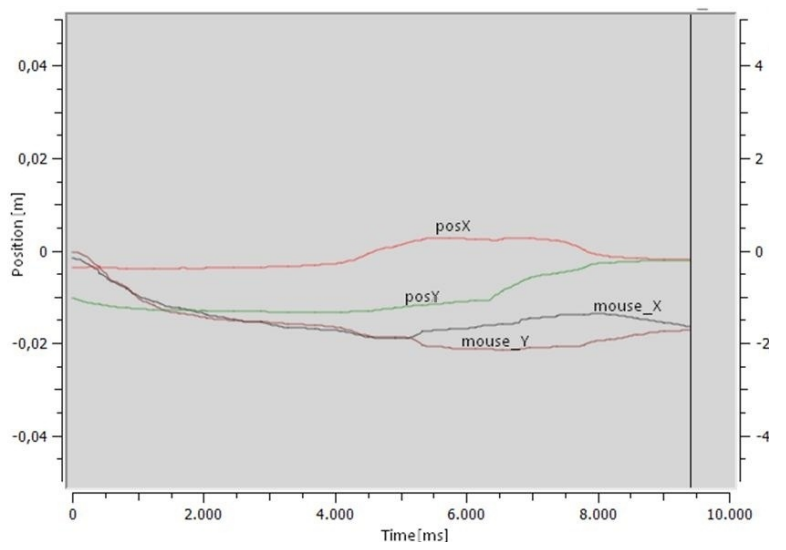

Figure 6: Motion Copying strategy. Mouse trajectory follows the trajectory on the haptic device in the same plane as indicated by the $\mathrm{x}$ and $\mathrm{y}$ axes.

Steering. Steering appeared to be a communication strategy that evolved from Motion Copying as a way to bypass the constraints of the limited workspace of the haptic device. 
Once in an area near the target location, the communication strategy was observed to switch from Motion Copying to steering during the homing-in phase.

The dyad members were using the steering strategy if:

a) Haptic device sequentially followed the axes.

b) Translational or PTR pointing motion inside the haptic device active workspace was observed, or rotational pointing motion in device workspace center was observed.

c) The beginning phase of the device motion in one direction expressed a velocity spike.

d) The stopping phase of the device motion pointing in one direction also expressed a velocity spike in an opposite direction.

e) Supervisor pointing in a direction was responded by acting agent movement along the same direction. Mouse movements were usually continuous for the first direction, and continuous or discrete for subsequent directions, after the first direction change.

f) A switch to Motion Copying strategy when close to the objective was done.

An example of Steering is shown in Figure 7.

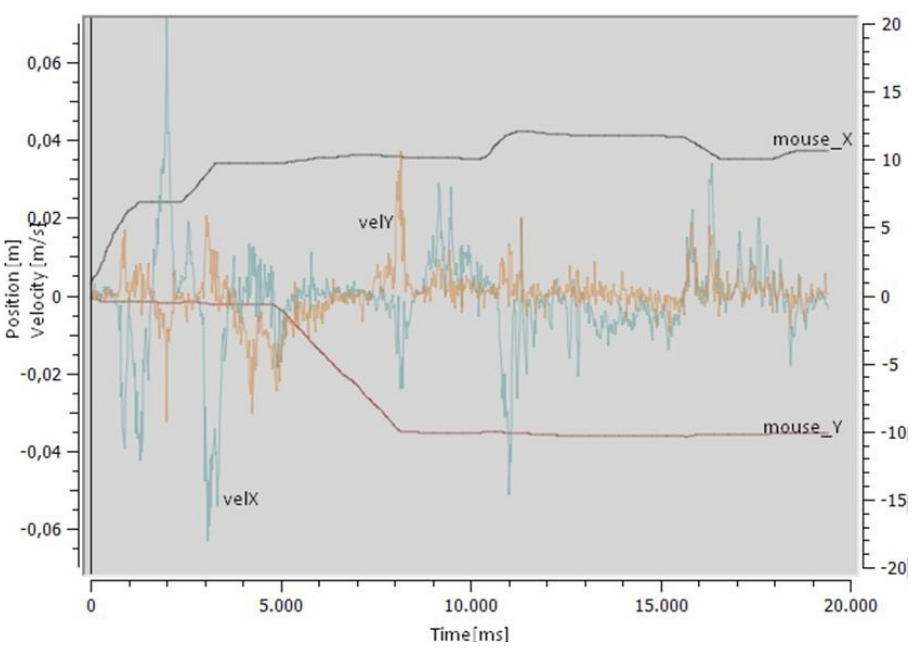

Figure 7: Steering strategy. First strong impulse by the haptic device in a certain direction sets the direction of the mouse movement. Subsequent impulse in the opposite direction by the haptic device stops the ongoing mouse movement.

Impulse Control. Impulse Control happened when the haptic device was displaced or rotated from the center of the workspace with a very high velocity in the desired direction, and then returned into the center with a lower velocity. Impulse Control communication strategy is described by the following characteristics:

a) Haptic device sequentially followed the axes.

b) Impulses could be produced by device translations, rotations or a mixture of both types of movements.

c) Haptic device workspace center was used for translational and rotational impulses. Active workspace was used when big PTR impulses were being applied.

d) The frequency of movement impulses was between 0.5 $\mathrm{Hz}$ and $2.5 \mathrm{~Hz}$. e) The mouse response was a series of discrete movements along the desired direction.

f) The Acting Agent used displacements of variable length to guess a position closer to the target. This is an indication of a predictive type of mouse control. Predictive types of mouse control was observed only in the impulse control strategy.

An example of Impulse Control is shown in Figure 8.

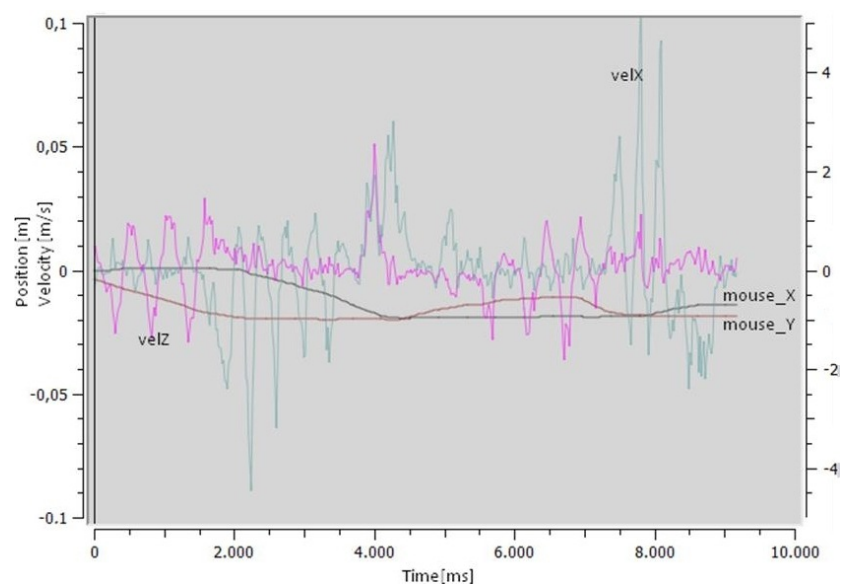

Figure 8: Impulse Control strategy. The mouse is moved in the direction of the impulses on the haptic device as long as the impulses persist.

\section{Verbal Communication Strategies}

Conversations between members in the dyad were transcribed from the videotapes and the transcripts were checked for regular patterns. Four strategies for verbal communication were identified.

Verbal Impulses. This strategy used only relative directional commands. Directional commands were repeated with a certain frequency, which usually depended on the cursor movement rate. If the cursor movement was slow and continuous, the frequency of verbal commands was high, and if the cursor movement was discrete with bigger steps, then the rate of verbal commands was smaller. It was either relative ("a bit further") or numerical (" $2 \mathrm{~cm}$ further").

Verbal Steering. This strategy also used relative directional commands. The movement was sequential along the axes of the workspace. Unlike the Verbal Impulses, only one directional command was given for the movement in a certain direction. This command was responded to by continuous mouse movements until the stopping command was announced. Then, the command for the next direction was given.

Accurate Positioning. This strategy was always used in combination with other verbal strategies to control the cursor in the area around the target point, or at the end of the long continuous movements along the axis. Relative directions with semantic quantification were dominantly (i.e. little more left, little bit). Commands were responded to with small discrete 
movements of the mouse. Stopping command was not strictly used.

General Positioning. This strategy was always used in combination with other verbal strategies. Verbal commands were based on the description of the position of the target point in the screen reference frame. Descriptive verbal commands were responded to with either slow continuous mouse movements in the desired direction, or large discrete steps. Usually, large movements of the cursor were terminated with the stopping command, after which Verbal Impulses or Accurate Positioning was applied.

\section{DISCUSSION}

In this study, a simple and classic Fitts' task was chosen to avoid possible confounding effects due to task characteristics. The effectiveness of communication modality on collaboration in a virtual $2 \mathrm{D}$ task space could then be examined. Task completion time results in the haptic communication condition conformed to the expected results of a Fitts' task, suggesting that meaningful and effective haptic communication between the members of the dyad was possible.

The effect of the order of introduction of modality into the experiment was unexpected. Results showed that collaboration using verbal communication was the most effective, and was independent of whether subjects had experience using the haptic modality. On the other hand, prior verbal communication experience had a negative transfer effect on performance in haptic communication. One explanation would be that since verbal is a more natural or commonly used mode of communication, what subjects learned about the task during the verbal condition actually interfered with the interpretation of haptic cues. So much so that it was better to have subjects start with the haptic communication if the goal was to maximize collaborative performance using haptic communication. In reality, this may be difficult to achieve as most people have had a lifetime's worth of experience communication verbally. Nevertheless, collaboration in the virtual environment may be relatively novel to most users that this learning strategy may still be effective.

The classification of the haptic communication strategies, with the developement of the appropriate taxonomy, was an attempt to answer the question of possible ways to transmit the data in collaborative tasks when using kinesthetic haptic devices. It was noted that some of the haptic strategies had their counterparts among the verbal strategies.

Motion Copying strategy was always used first in dyad trials, but was rarely effective. Some of the dyads never succeeded to evolve beyond that strategy. The ones who did, began to use either Impulse Control or Steering Strategy. After the initial period of familiarization with these strategies, dyads were able to achieve performance times comparable in level to verbal communication.
Research on haptic communication is still in its beginning. This experiment has shown that pairs of users were able to develop haptic communication strategies for information transmission in collaborative task and use these strategies to communicate effectively. This work showed a new approach to the research of haptic communication in which hand movements on the haptic device were analyzed in detail along with measured physical values. It also has implications for the design and implementation of haptics as a communication modality in collaborative virtual environments.

\section{ACKNOWLEDGEMENTS}

This work was supported in part by an award of Chaire Régionale de Chercheur Étranger de la Région Pays de la Loire. The authors are grateful to the grad students of Ecole des Mines de Nantes, and the EMARO students at the Institut de Recherche en Communications et Cybernétique de Nantes, France.

\section{REFERENCES}

Chan, A., MacLean, K., \& McGrenere, J. (2008). Designing Haptic Icons to Support Collaborative Turn-Taking. International Journal of Human-Computer Studies , 66, 333-355.

Chellali, A., Dumas, C., \& Milleville-Pennel, I. (2010). WYFIWIF: A Haptic Communication Paradigm for Collaborative Motor Skills Learning. Proceedings of the Web Virtual Reality and Three-Dimensional Worlds 2010. Freiburg.

Chellali, A., Milleville-Pennel, I., \& Dumas, C. (2008). Elaboration of common frame of reference in Collaborative Virtual Environment. ECCE 2008 European Conference on Cognitive Ergonomics. Madeira.

Enriquez, M., \& MacLean, K. (2003). The Hapticon Editor: A Tool in Support of Haptic Communication Research. Proc. of the 11th Annual Symposium on Haptic Interfaces for Virtual Environments and Teleoperator Systems. Los Angeles.

Ho, C., Basdogan, C., Slater, M., Durlach, N., \& Srinivasan, M. (1998). An Experiment on The Influence of Haptic Communication on the Sense of Being Together. British Telecom Workshop on Presence in Shared Virtual Environments. Ipswich.

Mac Kenzie, I. (1991). Fitts' law as a performance model in human-computer interaction. Toronto: University of Toronto.

Reed, K., Peshkin, M., Colgate, J., \& Patton, J. (2004). Initial Studies in Human-Robot-Human Interaction: Fitts' Law for Two People. Proc. of IEEE International Conference on Robotics and Automation (ICRA). New Orleans. 